\title{
Dual Beam Approach to Nano-Scale Defect Correction of High-End Photolithographic Masks
}

\author{
J.G. Marshman, A.A. Graupera, and M.A. Coy \\ FEI Company, One Corporation Way, Peabody, MA 01960
}

As the device geometries of today's leading edge semiconductors reach nano-scale levels, the technology required at each step of the process becomes much more demanding and more expensive. Photolithography is being pushed to its limits by these demands. Photolithographic masks are no exception, and with increasing costs of fabrication of high-end masks, the ability to repair defects on the mask is more crucial than ever.

Traditionally, masks were repaired with laser tools, but as device features continued to shrink below the micron level, higher resolution and precision were required. Focused Ion Beam (FIB) tools, incorporating a liquid metal gallium ion source, were developed to contend with these issues on the next generation of masks. FIB repairs have been very successful to date, dealing with defects on various mask types. The tools and the strategies used have continued to evolve to keep pace with the mask technology, and FIB tools are still being used successfully at the $65 \mathrm{~nm}$ node.

The next generation of masks will have even more stringent requirements in terms of feature size, transmission and CD specifications of the resulting printed circuit lines on the wafer. The inherent limitations of FIB repair are being realized, and higher resolution, increased precision and reduced residual damage are required. This demand has led to the development of a dual beam tool, combining an already successful FIB column with a field emission ESEM column. These complimentary technologies provide the optimum flexibility and number of repair strategies, with the FIB contributing well known and characterized repair capabilities and the ESEM allowing damage free scanning, charge control and providing a higher resolution process for current and future generation defects.

Additionally, because of the high level integration of the two columns, combination repair strategies have been developed, involving both columns, to provide the best possible repair results. Use of the FIB for rapid milling down through the bulk of the material, and the electron beam for the final material removal, allows for a fast process, with minimal residual damage to the underlying quartz substrate. This strategy eliminates the buildup of $\mathrm{Ga}$ ions implanted in the quartz, which can affect phase and transmission of the repaired area. A second combination strategy involves using the SEM to alter the electrical properties of the mask surface in order to improve image contrast and stability for an FIB repair. This is done by depositing a conductive layer (using a precursor chemistry with electron beam scanned in a selected area) only a few nanometers in thickness which can later be easily removed with no adverse effects.

Successful repairs strategies have been developed and demonstrated on a wide range of defect types, both opaque and clear, and on various mask types, including phase shift masks (PSM), Cr and others. These repairs have been analyzed with both CD metrology and Aerial Imaging Microscopy (AIMS) and have shown promising results 


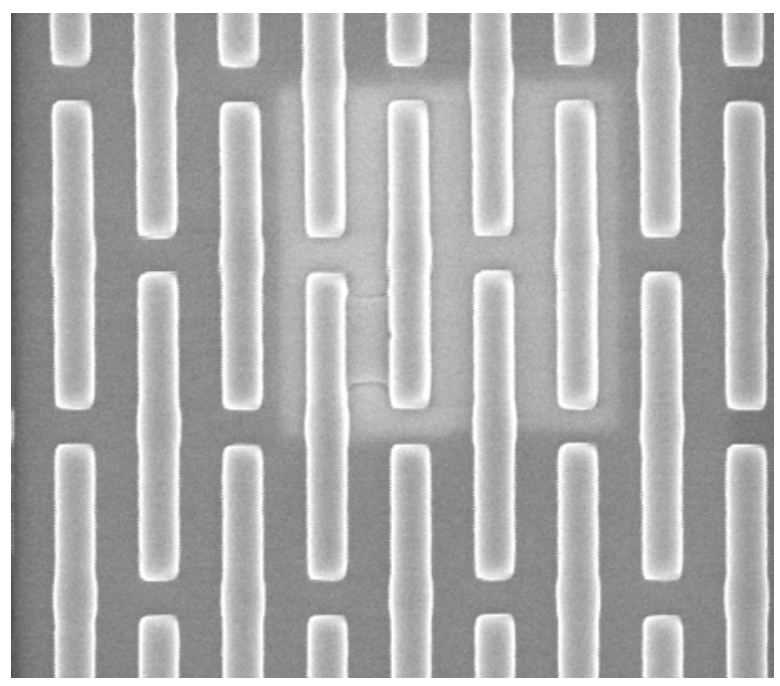

Figure 1: FIB repair of opaque bridge on a MoSi phase shift mask. Note the $\mathrm{Ga}+$ staining on the mask in the area surrounding the repair. $\mathrm{FOV}=8 \mu \mathrm{m}$

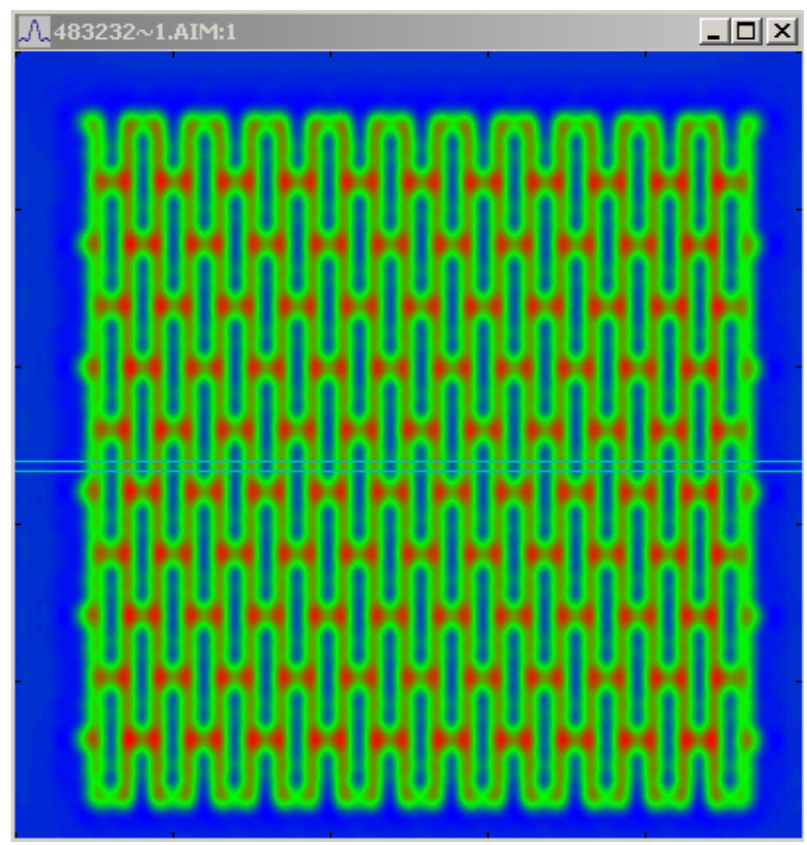

Figure 3: Aerial image of bridge repair in $193 \mathrm{~nm}$ transmitted light (Zeiss AIMSfab 193)

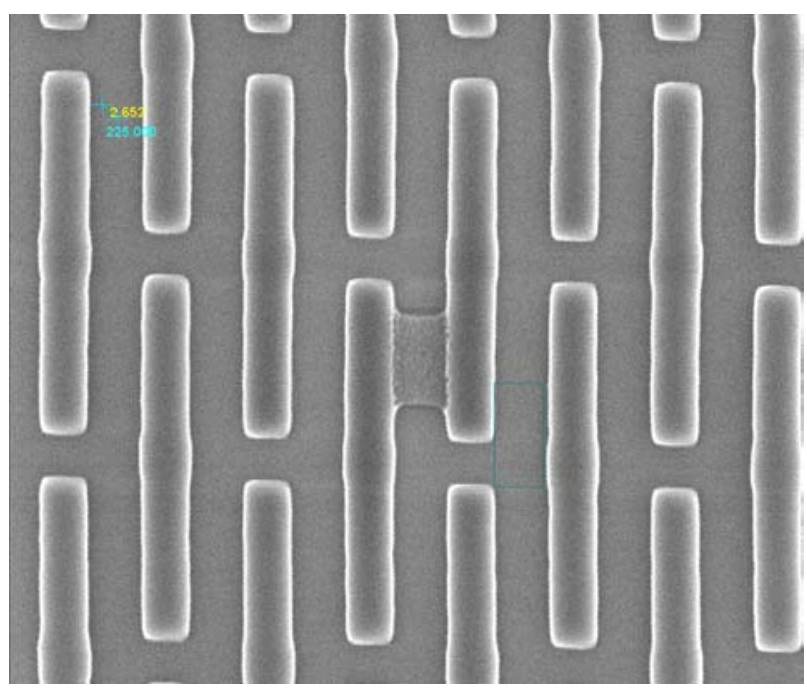

Figure 2: SEM repair of opaque bridge on MoSi phase shift mask. Electron beam repair leaves no residual damage on the mask. $\mathrm{FOV}=6.5 \mu \mathrm{m}$

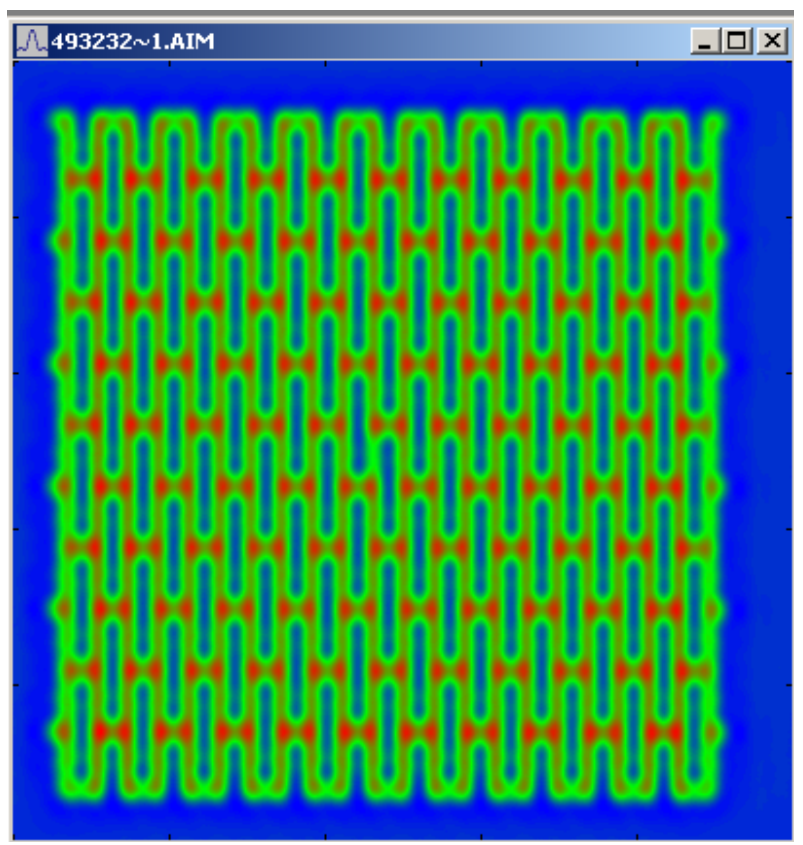

Figure 4: Aerial image of bridge defect in $193 \mathrm{~nm}$ transmitted light (Zeiss AIMSfab 193) 